\title{
Sizing a Serial Chain to Fit a Task Trajectory Using Clifford Algebra Exponentials*
}

\author{
Alba Perez \\ School of Engineering \\ Idaho State University \\ Pocatello, ID 83209, USA \\ perealba@isu.edu
}

\author{
J. Michael McCarthy \\ Robotics and Automation Laboratory \\ University of California, Irvine \\ Irvine, CA 92697, USA \\ jmmccart@uci.edu
}

\begin{abstract}
In this paper we formulate the "generalized inverse kinematics problem" for a spatial serial chain, where the goal is to determine values for structural parameters as well as for the joint parameters. The kinematics equations of the chain are formulated first using matrix exponentials and then cast into a form based on exponentials in a Clifford algebra. These equations contain the coordinates of the joint axes explicitly and have a systematic structure that can be exploited in their solution. As an example we fit a seven degree-of-freedom CCS chain to a 12 position task trajectory. In this problem, we can also specify desired values for the first two joint angles, and compute the structural parameters and the remaining joint angles.
\end{abstract}

\section{INTRODUCTION}

In this paper we consider the problem of computing the structural parameters for a serial chain, as well as its joint parameters, so the robot moves through task positions in a desired trajectory. This is a generalized version of the inverse kinematics problem, in which some or all of the structural parameters of the chain can be varied to change the properties of the robot. This has applications to modular and reconfigurable robotic systems (Moon and Kota [1], Yang and Chen [2]), as well as recovery planning for crippled robots.

The Clifford algebra exponential form of the kinematics equations is developed to provide a systematic set of design equations. These equations are directly related to the matrix exponential that is often used in robot kinematics, and have internal structure that can be exploited in their solution.

In what follows we derive the design equations for a serial chain constructed of $n$ cylindric joints, the $n \mathrm{C}$ chain, which specializes to serial chains consisting of revolute $(\mathrm{R})$ and prismatic $(\mathrm{P})$ joints $-\mathrm{R}$ denotes a revolute or hinged joint and $\mathrm{P}$ a prismatic or sliding joint. The complexity of this problem increases rapidly, and we show that the $5 R$ case involves the solution of 130 equations in 130 unknowns.

The use of these equations is demonstrated by an example design of a CCS serial chain to fit a task trajectory defined by 12 positions, in which we specify the angles of the first two joints and solve for the joint axes and the remaining joint values.

\footnotetext{
${ }^{*}$ This work is partially supported by NSF Grant
}

\section{LITERATURE REVIEW}

The geometric design of a robot manipulator defines the topology and dimensions of the articulated system that provides the end-effector position and velocity performance needed for a specified set of applications. Herve [3] shows how to use the subgroups of the Lie group of rigid body displacements to formulate robotic systems with desired workspace properties. Wenger [4] describes the benefits of new serial chain topologies that allow reconfiguration within the workspace. Chablat et al. [5] and $\mathrm{Li}$ et al. [6] demonstrate different approaches to the design of specialized parallel platforms that optimize position and velocity performance, which seeks a manipulator that has particular performance characteristics throughout its workspace.

A related approach to design seeks the robot manipulator that has specified position and velocity performance at precise locations within its workspace. Lee and Mavroidis [7], [8] formulate and solve the design equations for a 3R spatial chain that reaches four arbitrarily specified positions. This involves equating the kinematics equations of the chain to four selected positions and solving for the Denavit-Hartenberg parameters that satisfy these matrix equations. Perez and McCarthy [11] formulate and solve the design equations for the RRP and related chains. This work builds on a tradition of spatial mechanism synthesis dating back to Suh [9], and Tsai and Roth [10] - also see Suh and Radcliffe [12], and more recently McCarthy [13].

The complexity of the geometric design problem increases with the number of structural parameters. Four independent parameters define the axis of a revolute joint and two define a prismatic joint, therefore the spatial $5 \mathrm{R}$ chain has 20 structural parameters. Table I lists the five degree-of-freedom chains and their associated number $K$ of design parameters. It also lists the chains that are formed by combining the revolute and prismatic joints into spherical (S), cylindric (C) and universal (T) joints -an RRR chain with concurrent axes forms an S-joint, an RP chain with parallel axes forms a $\mathrm{C}$-joint, and an $\mathrm{RR}$ chain with perpendicular intersecting axes forms a T-joint. The design equations for the PPS, TS, CS, RPS, and RRS chains were originally formulated by Chen and Roth [14], and were recently solved by $\mathrm{Su}$ et al. [15] using polynomial homotopy. 


\begin{tabular}{|c|c|c|c|c|}
\hline Chain & Permut. & $\bar{K}$ & Special Cases & Permut. \\
\hline "RRPPP & $\overline{c 10}$ & $\overline{14}$ & $\begin{array}{l}\text { CCP } \\
\text { CRPP } \\
\text { TPPP }\end{array}$ & 19 \\
\hline RRRPP & 10 & 16 & $\begin{array}{l}\text { CCR } \\
\text { PPS } \\
\text { TCP } \\
\text { TRPP } \\
\text { CRRP }\end{array}$ & 36 \\
\hline RRRRP & 5 & 18 & $\begin{array}{l}\text { CS } \\
\text { RPS } \\
\text { TCR } \\
\text { TRRP } \\
\text { TTP } \\
\text { CRRR }\end{array}$ & 41 \\
\hline$\overline{\text { RRRRR }}$ & 1 & 20 & $\begin{array}{l}\text { TS } \\
\text { RRS } \\
\text { TTR } \\
\text { TRRR }\end{array}$ & 12 \\
\hline Total & $\overline{26}$ & & & 108 \\
\hline
\end{tabular}

TABLE I

THE FIVE DEGREE-OF-FREEDOM SERIAL CHAINS, A COUNT $K$ OF THE STRUCTURAL PARAMETERS, AND THE NUMBER OF PERMUTATIONS.

The goal of this paper is an efficient formulation of the design equations for these chains. A benefit of our approach is that it can also be applied to the design of serial chains with six or more degrees of freedom.

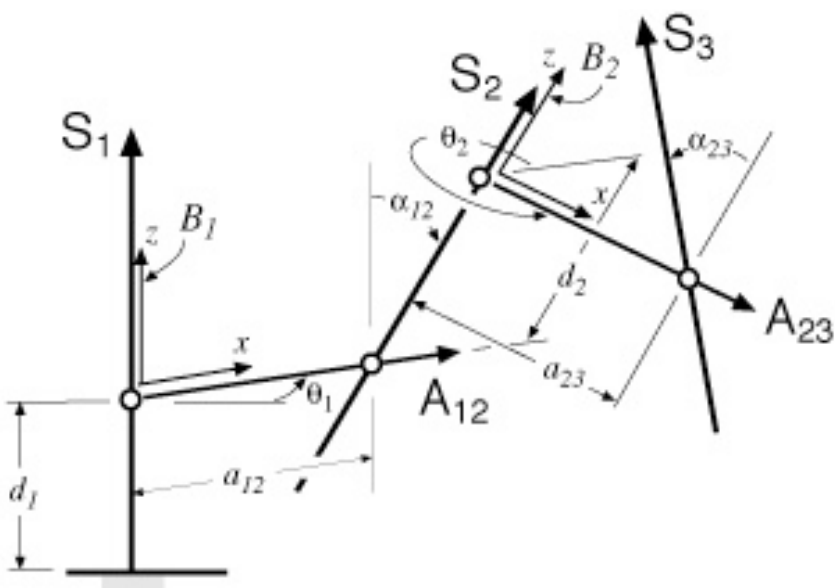

Fig. 1. Local frames for a serial chain.

\section{The Kinematics Equations of a Serial Chain}

The position and orientation of the end-effector of a serial chain is defined in terms of its joint parameters and physical dimensions by the kinematics equations. We use the Denavit-Hartenberg formulation to assign the local joint coordinate frames (Figure 1) necessary to define these equations, see Craig [16] and Tsai [17].

Let $S_{i}, i=1, \ldots n$, denote the $n$ joint axes in the chain, which may define the axis of either a revolute or a prismatic joint, respectively. Introduce the line $A_{i, i+1}$ which is the common normal to the axes $S_{i}$ and $S_{i+1}$. We now set the origin of the joint frame $T_{i}$ at the intersection of $\mathrm{S}_{i}$ and $\mathrm{A}_{i, i+1}$, such that the $\mathrm{z}$-axis is $\mathrm{S}_{i}$ and the $\mathrm{x}$-axis is $\mathrm{A}_{i, i+1}$, see McCarthy (1990)[18].

This allows us to write the kinematics equations of the chain in the form,

$$
\begin{aligned}
{[D]=} & {[G]\left[Z\left(\theta_{1}, d_{1}\right)\right]\left[X\left(\alpha_{12}, a_{12}\right)\right]\left[Z\left(\theta_{2}, d_{2}\right)\right] \ldots } \\
& {\left[X\left(\alpha_{n-1, n}, a_{n-1, n}\right)\right]\left[Z\left(\theta_{n}, d_{n}\right)\right][H], }
\end{aligned}
$$

where $\left[Z\left(\theta_{i}, d_{i}\right)\right]$ and $\left[X\left(\alpha_{i, i+1}, a_{i, i+1}\right)\right]$ are the $4 \times 4$ homogeneous transforms defining screw displacements about the $Z$-axis and about the $X$-axis respectively. The parameters $\theta_{i}$ and $d_{i}$ define the rotation of a revolute joint and slide of a prismatic joint, respectively, and $\alpha_{i, i+1}$ and $a_{i, i+1}$ define the dimensions of each link. Collectively they are known as the Denavit-Hartenberg parameters. The transformation $[G]$ locates the base of the robot in the world frame, and $[H]$ locates the tool frame relative to the last joint frame.

\section{A. Relative Displacements}

If we choose a reference position for the end-effector, denoted by $\left[D_{0}\right]$, called the zero reference position by Gupta (1986)[20], the displacement of the serial chain relative to this reference configuration is defined by $[D(\Delta \vec{\theta})]=$ $[D(\vec{\theta})]\left[D\left(\vec{\theta}_{0}\right)\right]^{-1}$. Assume that $\left[D_{0}\right]$ is a general position of the end-effector defined by joint parameters $\vec{\theta}_{0}$, so $\Delta \vec{\theta}=\vec{\theta}-\vec{\theta}_{0}$. Then, using the kinematics equations (1), we have

$$
\begin{aligned}
{[D(\Delta \vec{\theta})]=} & \left([G]\left[Z\left(\theta_{1}, d_{1}\right)\right] \ldots\left[Z\left(\theta_{n}, d_{n}\right)\right][H]\right) \\
& \left.\left([G]\left[Z\left(\theta_{10}, d_{10}\right)\right] \ldots\left[Z\left(\theta_{n_{0}}, d_{n_{0}}\right)\right][H]\right)\right)^{-1} .
\end{aligned}
$$

If we expand this expression, the relative transformation takes the form

$$
[D(\Delta \vec{\theta})]=\left[T\left(\Delta \theta_{1}, \mathrm{~S}_{1}\right)\right]\left[T\left(\Delta \theta_{2}, \mathrm{~S}_{2}\right)\right] \ldots\left[T\left(\Delta \theta_{n}, \mathrm{~S}_{n}\right)\right],
$$

where $S_{i}$ are the Plucker coordinates of each joint axis at the reference configuration expressed in the world frame.

\section{B. The Product of Exponentials}

Rather than use the Denavit-Hartenberg parameters for design, we write the $4 \times 4$ homogeneous transforms as matrix exponentials, (Murray et al.(1994)[19]) so the Plücker coordinates of the joint axes appear explicitly in the kinematics equations.

Consider a displacement defined such that the moving body rotates the angle $\phi$ and slides the distance $k$ around and along the screw axis $\mathbf{S}=(\mathbf{S}, \mathbf{C} \times \mathbf{S})$. Let $\mu=k / \phi$, then we can introduce the screw $\mathbf{J}=(\mathbf{S}, \mathbf{V})=(\mathbf{S}, \mathbf{C} \times \mathbf{S}+\mu \mathbf{S})$, where $\mu$ is called the pitch of the screw. The components of $\mathrm{J}$ define the $4 \times 4$ twist matrix,

$$
J=\left[\begin{array}{cccc}
0 & -s_{z} & s_{y} & v_{x} \\
s_{z} & 0 & -s_{x} & v_{y} \\
-s_{y} & s_{x} & 0 & v_{z} \\
0 & 0 & 0 & 0
\end{array}\right],
$$


and we find that the $4 \times 4$ homogeneous transform $[T(\phi, k, \mathrm{~S})]$ that performs a rotation of angle $\phi$ and a translation $k$ about and along the screw axis $\mathrm{S}$ is defined as the matrix exponential

$$
[T(\phi, k, \mathrm{~S})]=e^{\phi J}
$$

Using the exponential form the transformations $\left[T\left(\Delta \theta_{i}, \mathrm{~S}_{i}\right)\right]$, we obtain the relative kinematics equations (3) as

$$
[D(\Delta \vec{\theta})]=e^{\Delta \theta_{1} S_{1}} e^{\Delta \theta_{2} S_{2}} \ldots e^{\Delta \theta_{n} S_{n}} .
$$

The product of exponentials form of the kinematics equations (1) is now obtained as

$$
[D]=[D(\Delta \vec{\theta})]\left[D_{0}\right]=e^{\Delta \theta_{1} S_{1}} e^{\Delta \theta_{2} S_{2}} \ldots e^{\Delta \theta_{n} S_{n}}\left[D_{0}\right] .
$$

\section{The Even Clifford Algebra $C^{+}\left(P^{3}\right)$}

The Clifford algebra of projective three space $P^{3}$ is a sixteen-dimensional vector space with a product operation that is defined in terms of a scalar product, see McCarthy (1990)[18]. The elements of even rank form an eight dimensional subalgebra $C^{+}\left(P^{3}\right)$ that can be identified with the set of $4 \times 4$ homogeneous transforms. Mullineux [21] describes the use of this Clifford algebra for motion interpolation, and Perez and McCarthy [23] and Daniilidis [22] describe its use in robot design and camera calibration, respectively. See also Castelain [25].

The typical element of $C^{+}\left(P^{3}\right)$ can be written as the eight dimensional vector given by

$$
\hat{A}=a_{0}+a_{1} i+a_{2} j+a_{3} k+a_{4} \epsilon+a_{5} i \epsilon+a_{6} j \epsilon+a_{7} k \epsilon,
$$

where the basis elements $i, j$, and $k$ are the well-known quaternion units, and $\epsilon$ is called the dual unit. The dual number $\epsilon$ commutes with $i, j$, and $k$, and multiplies by the rule $\epsilon^{2}=0$.

In our calculations, we use the notation $\mathbf{A}=a_{1} i+a_{2} j+$ $a_{3} k$ and $\mathbf{A}^{\circ}=a_{5} i+a_{6} j+a_{7} k$-the small circle superscript is often used to distinguish coefficients of the dual unit. This allows us to write the Clifford algebra element (8) as

$$
\hat{A}=a_{0}+\mathbf{A}+a_{4} \epsilon+\mathbf{A}^{\circ} \epsilon .
$$

Now, collect the scalar and vector terms so this element takes the form of a dual vector and a dual number,

$$
\hat{A}=\left(a_{0}+a_{4} \epsilon\right)+\left(\mathbf{A}+\mathbf{A}^{\circ} \epsilon\right)=\hat{a}+\mathbf{A} .
$$

Using this notation the Clifford algebra product of elements $\hat{A}=\hat{a}+\mathrm{A}$ and $\hat{B}=\hat{b}+\mathrm{B}$ takes the form

$$
\hat{C}=(\hat{b}+\mathrm{B})(\hat{a}+\mathrm{A})=(\hat{b} \hat{a}-\mathrm{B} \cdot \mathrm{A})+(\hat{a} \mathrm{~B}+\hat{b} \mathrm{~A}+\mathrm{B} \times \mathrm{A}),
$$

where the usual vector dot and cross products are extended linearly to dual vectors.

\section{A. Exponential of a screw}

The Plücker coordinates $\mathbf{S}=(\mathbf{S}, \mathbf{C} \times \mathbf{S})$ of a line are given by a dual vector that represents the direction and location of the line. The first vector $\mathbf{S}$ gives the direction, while the second vector $\mathbf{C} \times \mathbf{S}$ is constructed as the cross product of a point on the line times the direction, and it is called the moment of the line. These can be identified with the Clifford algebra element $\mathbf{S}=\mathbf{S}+\epsilon \mathbf{C} \times \mathbf{S}$. Similarly, the screw $\mathrm{J}=(\mathbf{S}, \mathbf{V})=(\mathbf{S}, \mathbf{C} \times \mathbf{S}+\mu \mathbf{S})$ becomes the element $\mathbf{J}=\mathbf{S}+\epsilon \mathbf{V}=(1+\mu \epsilon) \mathbf{S}$. Using the Clifford product we can compute the exponential of the screw $\theta \mathrm{J}$,

$$
e^{\theta \mathrm{J}}=1+\mathrm{J}+\frac{\theta^{2}}{2} \mathrm{~J}^{2}+\frac{\theta^{3}}{3 !} \mathrm{J}^{3}+\cdots .
$$

to obtain

$$
\begin{aligned}
e^{\theta \mathrm{J}} & =(\cos \theta-d \sin \theta \epsilon)+(\sin \theta+d \cos \theta \epsilon) \mathrm{S} \\
& =\cos \hat{\theta}+\sin \hat{\theta} \mathrm{S} .
\end{aligned}
$$

with the dual angle $\hat{\theta}=\theta+d \epsilon$.

Equation in (13) introduces the unit dual quaterion which is identified with spatial displacements,

$$
\hat{Q}=\cos \frac{\hat{\phi}}{2}+\sin \frac{\hat{\phi}}{2} \mathbf{S}=\left(1+\frac{1}{2} \mathbf{d} \epsilon\right)\left(\cos \frac{\phi}{2}+\sin \frac{\phi}{2} \mathbf{S}\right),
$$

where the rotation angle is $\phi=2 \theta$.

\section{B. Clifford algebra kinematics equations}

The composition of Clifford algebra elements defines the relative kinematics equations for a serial chain that are equivalent to (6).

Consider the $n \mathrm{C}$ serial chain in which each joint can rotate an angle $\theta_{i}$ around, and slide the distance $d_{i}$ along, the axis $\mathrm{S}_{i}$, for $i=1, \ldots, n$. Let $\vec{\theta}_{0}$ and $\overrightarrow{d_{0}}$ be the joint parameters of this chain when it is in its reference configuration, so we have

$$
\Delta \hat{\vec{\theta}}=(\vec{\theta}+\vec{d} \epsilon)-\left(\vec{\theta}_{0}+\vec{d}_{0} \epsilon\right)=\left(\Delta \hat{\theta}_{1}, \Delta \hat{\theta}_{2}, \ldots, \Delta \hat{\theta}_{n}\right) .
$$

Then, the movement from this reference configuration is defined by the kinematics equation,

$$
\begin{aligned}
\hat{D}(\Delta \hat{\vec{\theta}})= & e^{\frac{\Delta \hat{\theta}_{1}}{2} \mathrm{~S}_{1}} e^{\frac{\Delta \hat{\theta}_{2}}{2} \mathrm{~S}_{2}} \cdots e^{\frac{\Delta \hat{\theta}_{n}}{2} \mathrm{~S}_{n}}, \\
= & \left(\mathrm{c} \frac{\Delta \hat{\theta}_{1}}{2}+\mathrm{s} \frac{\Delta \hat{\theta}_{1}}{2} \mathrm{~S}_{1}\right)\left(\mathrm{c} \frac{\Delta \hat{\theta}_{2}}{2}+\mathrm{s} \frac{\Delta \hat{\theta}_{2}}{2} \mathrm{~S}_{2}\right) \cdots \\
& \left(\mathrm{c} \frac{\Delta \hat{\theta}_{n}}{2}+\mathrm{s} \frac{\Delta \hat{\theta}_{n}}{2} \mathrm{~S}_{n}\right) .
\end{aligned}
$$

Note $\mathrm{s}$ and $\mathrm{c}$ denote the sine and cosine functions, respectively.

\section{Design Equations for a Serial Chain}

The goal of our design problem is to determine the dimensions of a spatial serial chain that can position a tool held by its end-effector in a given set of task positions. The location of the base of the robot, the position of the tool frame, as well as the link dimensions and joint angles are considered to be design variables. 


\section{A. Specified task positions}

Identify a set of planar task positions $\left[P_{j}\right], j=1, \ldots, m$. Then, the physical dimensions of the chain are defined by the requirement that for each position $\left[P_{j}\right]$ there is a joint parameter vector $\vec{\theta}_{j}$ such that the kinematics equations of the chain satisfy the relations

$$
\left[P_{j}\right]=\left[D\left(\vec{\theta}_{j}\right)\right], \quad i=1, \ldots, m .
$$

Now, choose $\left[P_{1}\right]$ as the reference position and compute the relative displacements $\left[P_{j}\right]\left[P_{1}^{-1}\right]=\left[P_{1 j}\right], j=2, \ldots, m$.

For each of these relative displacements $\left[P_{1 j}\right]$ we can determine the dual unit quaternion $\hat{P}_{1 j}=\cos \frac{\hat{\phi}_{1 j}}{2}+\sin \frac{\hat{\phi}_{1 j}}{2} \mathrm{P}_{1 j}$, $j=2, \ldots, m$. Now writing equation (16) for the $m-1$ relative displacements, we obtain

$$
\hat{P}_{1 j}=e^{\frac{\Delta \hat{\theta}_{1 j}}{2} S_{1}} e^{\frac{\Delta \hat{\theta}_{2 j}}{2} S_{2}} \ldots e^{\frac{\Delta \hat{\theta}_{n j}}{2} S_{n}}, \quad j=2, \ldots, m .
$$

The result is $8(m-1)$ design equations. The unknowns are the $n$ joint axes $\mathrm{S}_{i}, i=1, \ldots, n$, and the $n(m-1)$ pairs of joint parameters $\theta_{i j}+d_{i j} \epsilon$.

\section{B. Counting}

The eight components of the unit Clifford algebra kinematics equations (18) are not independent. In particular, it is easy to see that a dual unit quaternion satisfies the identity,

$$
\hat{Q} \hat{Q}^{*}=e^{\frac{\Delta \hat{\phi}}{2} \mathrm{~S}} e^{-\frac{\Delta \hat{\phi}}{2} \mathrm{~S}}=1,
$$

which imposes a pair of constraints. This means that we have 6(n-1) independent design equations.

We now count the joint axis parameters in a chain that consists of $r$ revolute joints and $p$ prismatic joints. A prismatic joint is defined by the two parameters of the unit vector $\mathbf{S}$ that defines the slide direction. The revolute joint axis is defined by Plücker coordinate vectors, $\mathrm{S}_{i}=\mathbf{S}+\mathbf{C} \times \mathbf{S} \epsilon$, that have four independent components.

Thus, the joint axes that define this chain have $4 r+2 p$ independent unknowns. In addition, there are $(r+p)(m-$ 1) unknown joint parameters that define the $m$ relative positions. We obtain

$$
\begin{aligned}
E & =4 r+2 p+(r+p)(m-1)-6(m-1) \\
& =(3 r+p+6)+(r+p-6) m,
\end{aligned}
$$

where $E$ excess of unknowns over equations. This excess can be made to equal zero for chains with degree of freedom dof $=r+p \leq 5$, in which case we specify

$$
m=\frac{3 r+p+6}{6-r-p},
$$

task positions. If fewer than this number of task positions are defined, or if the chain has six or more degrees of free$\mathrm{dom}$, then we are free to select values for the excess design parameters. In Table II we show the basic five-degree-offreedom chains, the minimum number of parameters and equations, and the number of task positions that can be defined in order to obtain a finite set of solutions.

\begin{tabular}{|c|c|c|c|}
\hline Chain & $\mathrm{K}$ & Task positions & Total equations \\
\hline \hline RRPPP & 14 & 15 & 84 \\
\hline RRRPP & 16 & 17 & 96 \\
\hline RRRRP & 18 & 19 & 108 \\
\hline RRRRR & 20 & 21 & 120 \\
\hline \hline
\end{tabular}

TABLE II

THE NUMBER OF TASK POSITIONS THAT DETERMINE THE STRUCTURAL PARAMETERS FOR FIVE DEGREE-OF-FREEDOM SERIAL CHAINS.

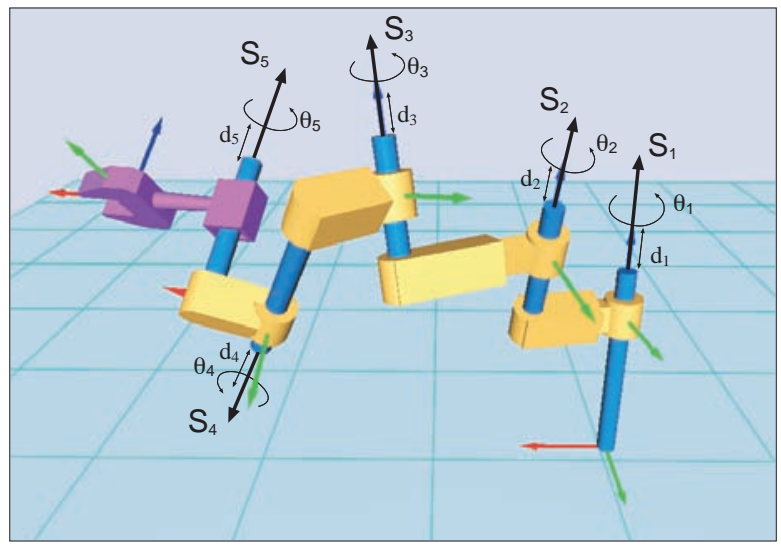

Fig. 2. The $5 \mathrm{C}$ serial robot.

\section{Assembling the Design Equations}

The structure of the Clifford algebra design equations provides a systematic approach to assembling the design equations for a broad range of serial chains. The basic approach is to formulate the design equations for the $n \mathrm{C}$ serial chain, and then i) restrict the joint variables to form prismatic or sliding joints, and ii) impose geometric conditions on the axes to form other joints. The result is a systematic way of defining the design equations for a broad range of chains. Here we present the procedure for the $2 \mathrm{C}$ serial chain, but it has been implemented for the $3 \mathrm{C}, 4 \mathrm{C}$ and $5 \mathrm{C}$ cases, as well. Figure 2 presents the $5 \mathrm{C}$ robot.

\section{A. The $2 C$ chain}

The Clifford algebra form of the relative kinematics equations for the $2 \mathrm{C}$ chain can be written as

$$
\hat{D}(\Delta \hat{\vec{\theta}})=\left(\mathrm{c} \frac{\Delta \hat{\theta}_{1}}{2}+\mathrm{s} \frac{\Delta \hat{\theta}_{1}}{2} \mathrm{~S}_{1}\right)\left(\mathrm{c} \frac{\Delta \hat{\theta}_{2}}{2}+\mathrm{s} \frac{\Delta \hat{\theta}_{2}}{2} \mathrm{~S}_{2}\right),
$$

where $\mathbf{S}_{i}=\mathbf{S}_{i}+\mathbf{S}_{i}^{\circ} \epsilon$ define the joint axes in the reference position, and $\Delta \hat{\theta}_{i}=\Delta \theta_{i}+\Delta d_{i}$ define the rotation and slide of the cylindric joint around the $i^{\text {th }}$ axis.

Expand the right side of (22) using the Clifford product to obtain

$$
\begin{aligned}
\hat{D}(\Delta \hat{\vec{\theta}})= & \hat{\mathrm{c}}_{1} \hat{\mathrm{c}}_{2}-\hat{\mathrm{s}}_{1} \hat{\mathrm{s}}_{2} \mathrm{~S}_{1} \cdot \mathrm{S}_{2}+ \\
& \hat{\mathrm{s}}_{1} \hat{\mathrm{c}}_{2} \mathrm{~S}_{1}+\hat{\mathrm{c}}_{1} \hat{\mathrm{s}}_{2} \mathrm{~S}_{2}+\hat{\mathrm{s}}_{1} \hat{\mathrm{s}}_{2} \mathrm{~S}_{1} \times \mathrm{S}_{2} .
\end{aligned}
$$

For convenience, we have introduced the notation $\hat{\mathrm{c}}_{i}=$ $\cos \frac{\Delta \hat{\theta}_{i}}{2}$ and $\hat{s}_{i}=\cos \frac{\Delta \hat{\theta}_{i}}{2}$. 
Equation (23) can be written in matrix form to emphasize that it is the linear combination of four monomials, which we assemble into an array in reversed lexicographic order obtained by reading right to left,

$$
\hat{\mathbf{V}}=\left(\hat{\mathrm{c}}_{1} \hat{\mathrm{c}}_{2}, \hat{\mathrm{s}}_{1} \hat{\mathrm{c}}_{2}, \hat{\mathrm{c}}_{1} \hat{\mathrm{s}}_{2}, \hat{\mathrm{s}}_{1} \hat{\mathrm{s}}_{2}\right)^{T} .
$$

Collecting terms in (23), we obtain the matrix equation

$$
\hat{\mathbf{D}}(\Delta \hat{\vec{\theta}})=\left[\begin{array}{cccc}
0 & \mathrm{~S}_{1} & \mathrm{~S}_{2} & \mathrm{~S}_{1} \times \mathrm{S}_{1} \\
1 & 0 & 0 & -\mathrm{S}_{1} \cdot \mathrm{S}_{2}
\end{array}\right] \hat{\mathbf{V}}
$$

The Clifford algebra notation is compact in that each column of this matrix actually forms a column of eight real coefficients. Similarly, each of the monomials in $\hat{\mathbf{V}}$ expands into four real terms, which we can list as

$$
\mathbf{M}=\left(\mathbf{V}, \frac{\Delta d_{1}}{2} \mathbf{V}, \frac{\Delta d_{2}}{2} \mathbf{V}\right),
$$

where $\mathbf{V}$ is the array of real parts of $\hat{\mathbf{V}}$. Thus, (25) expands to an $8 \times 12$ matrix equation. The number $k$ of joint variable monomials in an $n \mathrm{C}$ serial chain is given by

$$
k=(n+1) 2^{n} .
$$

Thus, these equations become $8 \times 32,8 \times 80$ and $8 \times 192$ for $3 \mathrm{C}, 4 \mathrm{C}$ and $5 \mathrm{C}$ chains.

The kinematics equations (25) can be used directly for the design of a $2 \mathrm{C}$ chain. They can be also specialized to obtain design equations for other chains.

\section{B. Special Cases}

The $i^{t h}$ cylindric joint in the $2 \mathrm{C}$ chain is converted to a revolute joint simply by setting $\Delta d_{i}=0$. The monomials in (26) that define the RC, RR or PP chains are given by

$$
\begin{array}{ll}
\mathrm{RC}: & \mathbf{M}=\left(\mathbf{V}, \frac{\Delta d_{2}}{2} \mathbf{V}\right), \\
\mathrm{RR}: & \mathbf{M}=\mathbf{V}, \\
\mathrm{PP}: & \mathbf{M}=\left(1, \frac{\Delta d_{1}}{2}, \frac{\Delta d_{2}}{2}\right) .
\end{array}
$$

The RR and RRR chains can be further specialized by introducing geometric constraints between their joint axes.

Consider the RR chain formed by axes $S_{i}$ and $S_{i+1}$. If we require these axes to intersect in a right angle, then we obtain Hooke's joint, denoted by a $\mathrm{T}$ following Crane and Duffy [26]. If the axes of an RRR chain are constrained so that they are perpendicular and intersect in a single point, then the chain allows full orientation freedom around this point, and is called a spherical, or S, joint.

\section{The Synthesis Algorithm}

The described approach has been used to create a general synthesizer algorithm for robots with two to five joints (Figure 2), allowing the design of robots from 2 to 10 degrees of freedom.

\section{A. Structure}

The general syntesizer contains the terms of the Clifford algebra kinematics equations for the $5 \mathrm{C}$ robot. In order to improve the calculations, the kinematics equations of the $5 \mathrm{C}$ robot are collected as a function of the monomials following the methodology introduced in Eq.(26). The 5C robot has 10 joint angles and the kinematics equations can be collected in 192 terms.

These terms are the 32 terms containing products of sines and cosines of the $\theta_{i}$ joint angles,

$$
\begin{aligned}
\mathbf{V}= & \left\{s_{1} s_{2} s_{3} s_{4} s_{5},\left(s_{1} s_{2} s_{3} s_{4} c_{5}\right)_{5},\left(s_{1} s_{2} s_{3} c_{4} c_{5}\right)_{10},\right. \\
& \left.\left(s_{1} s_{2} c_{3} c_{4} c_{5}\right)_{10},\left(s_{1} c_{2} c_{3} c_{4} c_{5}\right)_{5}, c_{1} c_{2} c_{3} c_{4} c_{5}\right\}
\end{aligned}
$$

plus 5 sets of terms containing the same terms multiplied by one of the joint slides $d_{i}$, to obtain the set of monomials,

$$
\mathbf{M}=\left\{\mathbf{V}, \frac{\Delta d_{1}}{2} \mathbf{V}, \frac{\Delta d_{2}}{2} \mathbf{V}, \frac{\Delta d_{3}}{2} \mathbf{V}, \frac{\Delta d_{4}}{2} \mathbf{V}, \frac{\Delta d_{5}}{2} \mathbf{V}\right\}
$$

The kinematics equations of the $5 \mathrm{C}$ robot can now be written as the sum of products,

$$
\hat{Q}_{5 C}=\sum_{i=1}^{192} \mathbf{K}_{i} M_{i}, \quad M_{i} \in \mathbf{M},
$$

where the 8-dimensional column vectors $\mathbf{K}_{i}$ contain the structural variables of the joint axes.

Any serial robot with up to 5 joints consisting of revolute $(\mathrm{R})$, prismatic $(\mathrm{P})$, universal $(\mathrm{T})$, cylindrical $(\mathrm{C})$ or spherical (S) joints can be modeled from the $5 \mathrm{C}$ robot by setting some of the joint variables and adding extra constraints. Notice that the $\mathrm{S}$ joint is modeled as three perpendicular axes whose directions can be arbitrarly chosen [24] and that intersect at a point. As an example, consider the kinematics equations obtained from the $5 \mathrm{C}$ general robot for the RPS robot:

$$
\hat{Q}_{R P S}=\hat{S}_{1}\left(\theta_{1}, 0\right) \hat{S}_{2}\left(0, d_{2}\right) \hat{X}\left(\theta_{3}, 0\right) \hat{Y}\left(\theta_{4}, 0\right) \hat{Z}\left(\theta_{5}, 0\right)
$$

The general synthesizer selects, from the kinematics equations of the $5 \mathrm{C}$ robot, those terms that are not zero for the desired robot topology. To do so, we identify the terms corresponding to a prismatic or a revolute joint, for each one of the joint axes $j$, that is, we define the list of indices $L_{R j}, L_{P j}$ and $L_{C j}$,

$$
\begin{aligned}
& L_{R j}=\left\{i \quad: \quad \cos \frac{\theta_{j}}{2} \in M_{i} \& \sin \frac{\theta_{j}}{2} \in M_{i} \& d_{j} \notin M_{i}\right\}, \\
& L_{P j}=\left\{i \quad: \quad d_{j} \in M_{i} \& \cos \frac{\theta_{j}}{2} \in M_{i} \& \sin \frac{\theta_{j}}{2} \notin M_{i}\right\} \text {, } \\
& L_{C j}=\left\{i \quad: \quad d_{j} \in M_{i} \& \cos \frac{\theta_{j}}{2} \in M_{i} \& \sin \frac{\theta_{j}}{2} \in M_{i}\right\} \text {, } \\
& j=1, \ldots 5 \text {. }
\end{aligned}
$$

The set of indices $L$ for the chosen topology is created by intersecting the indices corresponding to each one of the joints,

$$
L=\bigcap_{j=1}^{5} L_{(R, P, C) j},
$$


and the kinematics equations are constructed from this set of indices,

$$
\hat{Q}=\sum_{i \in L} \mathbf{K}_{i} M_{i}
$$

To create the design equations, we equate the kinematics equations (35) to the set of task positions $\hat{P}_{i}$ in dual quaternion form,

$$
\hat{Q}=\hat{P}_{i}, \quad, i=2, \ldots n,
$$

where $n$ is defined for the chosen topology based on the counting and the conditions imposed in the joint variables.

\section{B. Implementation}

The general solver has been implemented in Java for the cases of serial $5 \mathrm{C}, 4 \mathrm{C}, 3 \mathrm{C}$ and $2 \mathrm{C}$ serial robots, and their particular cases. A Levenberg-Marquardt numerical solver is used to compute the numerical solution. The algorithm is a Java translation by Steve Verrill from the FORTRAN MINPACK source produced by Garbow, Hillstrom, and others.

\section{APPLICATION EXAMPLE}

We have chosen twelve positions from a trajectory generated using a Bezier interpolation consisting of three intermediate positions between each two key positions. The 12 positions shown in Figure 3 define the task.

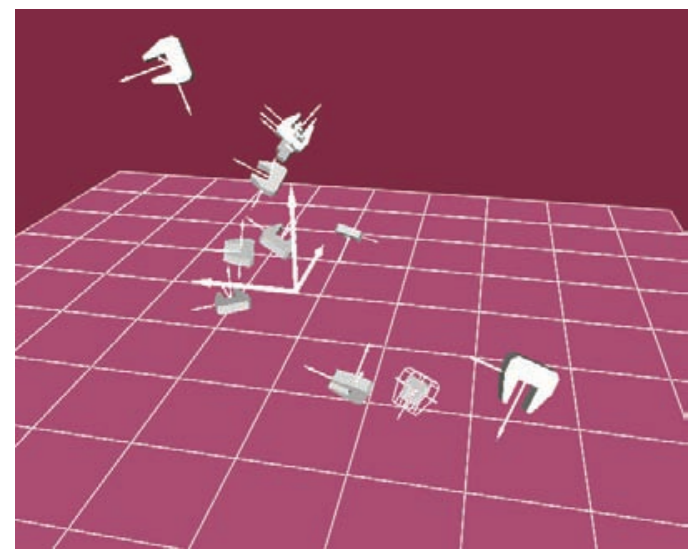

Fig. 3. The 12 positions defining the task

As an example, we have chosen to design a 7-degreeof-freedom CCS serial robot. It consists of a shoulder and elbow that allow both rotation and translation about and along the axes, and a spherical wrist; see Figure 4.

In addition, we want a design so that the first joint of the robot moves along the task positions avoiding big displacements of the robot. To accomplish this, we impose that the resulting design must have certain values in the rotation and translation of the first joint for each of the task positions. See Table III for the task positions and the angle conditions.

For this example, we run the Java software twice to obtain two different solutions. The first solution took two iterations of the solver with a total time of 91 seconds. The second solution took 353 seconds and three iterations. See

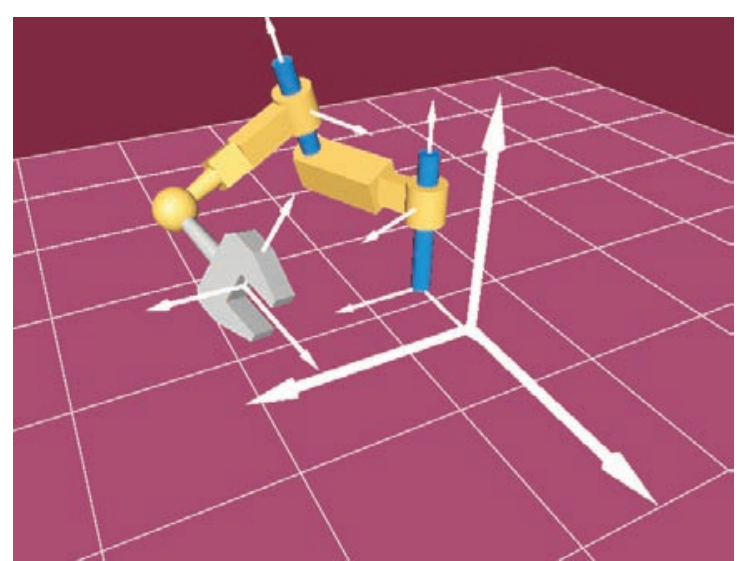

Fig. 4. The CCS serial robot

\begin{tabular}{lc}
\hline Task & Dual quaternion coordinates \\
\hline 1 & $(0.02,-0.15,0.58,0.80,0.66,-0.30,0.09,-0.14)$ \\
2 & $(0.06,-0.37,0.38,0.85,0.77,-0.38,0.20,-0.31)$ \\
3 & $(-0.03,-0.44,-0.90,0.03,-0.38,0.77,-0.34,0.67)$ \\
4 & $(0.41,-0.29,-0.41,0.76,0.32,0.37,0.14,0.04)$ \\
5 & $(-0.04,-0.43,-0.90,0.05,-0.39,0.74,-0.30,0.64)$ \\
6 & $(0.28,-0.01,-0.36,0.89,-0.07,-0.06,0.11,0.06)$ \\
7 & $(0.36,0.35,0.48,0.72,-0.43,-0.54,-0.12,0.56)$ \\
8 & $(-0.18,0.79,0.03,0.58,-0.73,-0.94,-0.51,1.07)$ \\
9 & $(-0.29,-0.54,0.55,0.57,0.87,-0.26,1.106,-0.87)$ \\
10 & $(-0.93,-0.19,0.26,0.19,0.27,-0.07,0.10,1.09)$ \\
11 & Values \\
\hline Joint var. & $i \pi / 11, \quad i=1 \ldots 11$ \\
\hline$\theta_{1}$ & $0.2 i, \quad i=1 \ldots 11$ \\
$d_{1}$ & TABL
\end{tabular}

TABLE III

TASK POSITIONS AND VALUES FOR THE FIRST JOINT ANGLES

Table IV for the coordinates of the joint axes. Figure 5 shows the resulting robots moving along the desired task.

\begin{tabular}{lrr}
\hline Sol. & Joint Axis & Value \\
\hline 1 & 1 & $(0.14,0.49,0.86,-3.59,-0.45,0.85)$ \\
& 2 & $(-0.24,0.92,0.29,-2.44,-0.73,0.26)$ \\
& wrist & $(0.23,1.21,-0.12)$ \\
\hline 2 & 1 & $(0.72,-0.50,0.48,0.40,0.03,-0.57)$ \\
& 2 & $(-0.10,0.52,-0.85,-2.53,0.17,0.41)$ \\
& wrist & $(0.05,0.72,-0.94)$ \\
\hline
\end{tabular}

TABLE IV

CCS ROBOTS DESIGNED TO PERFORM THE SPECIFIED TASK.

\section{SUMmary}

This paper uses the kinematics equations of a spatial $n \mathrm{C}$ chain to formulate the generalized inverse kinematics problem. Rather than simply compute the joint angles of a robot for a given task trajectory, we also seek the structural parameters. This is a design problem with applications to modular and reconfigurable robotic systems. This approach may also be useful in the calibration of robots.

The exponential form of the kinematics equations of the chain are reformulated using Clifford algebra to obtain an efficient and systematic set of equations. We automated 


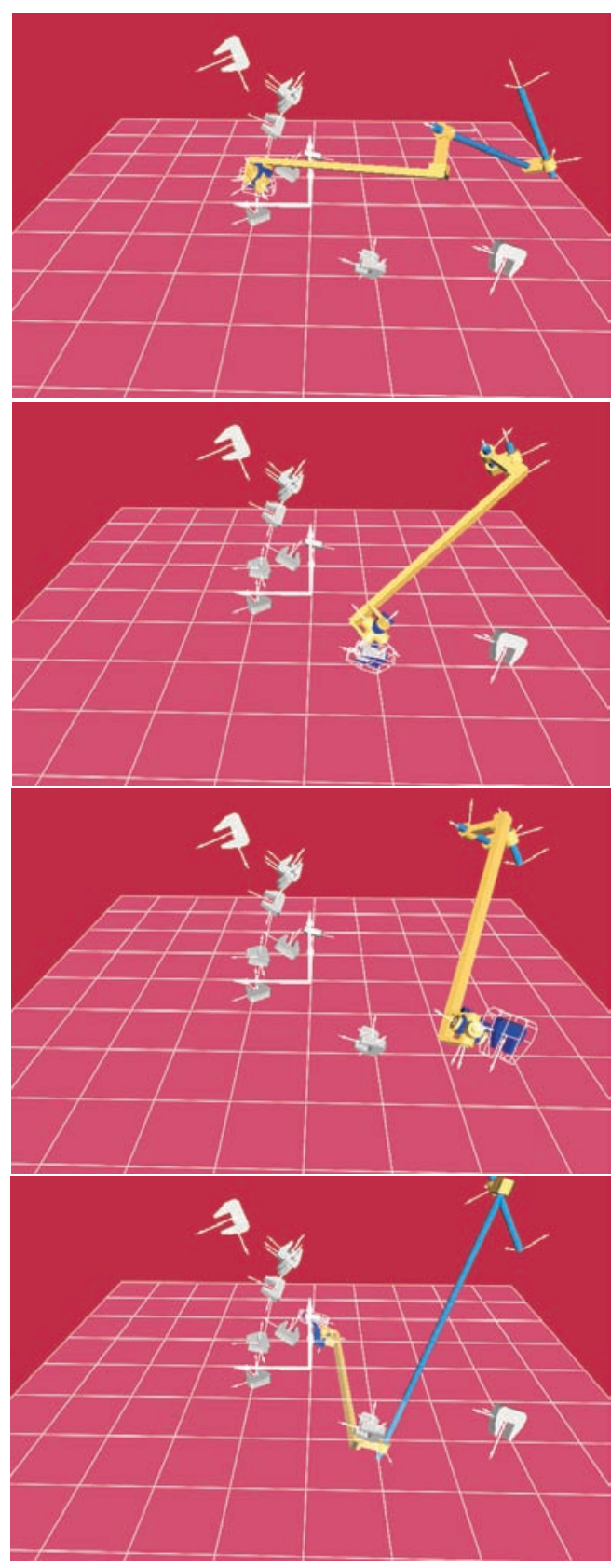

Fig. 5. Solution 1 CCS robot

the derivation of the design equations for robots derived from $2 \mathrm{C}$ to $5 \mathrm{C}$ chains, and verified them using a numerical solver.

These generalized inverse kinematics problems become complicated rapidly. In particular, fitting a $5 \mathrm{R}$ chain to a 21 task trajectory requires the solution of 130 equations in 130 unknowns. While we can obtain individual solutions numerically, a bound on the total number of chains that can fit a given task is unknown at this time.

We demonstrate the solution of this problem by determining the structural parameters of a CCS serial chain so that it reaches an arbitrarily specified 12 position task trajectory. In this problem we are free to specify values for the first two joint parameters, and solve for the remaining.

\section{REFERENCES}

[1] Moon, Y-M, and Kota, S, 2002, "Generalized Kinematic Modeling of Reconfigurable Machine Tools," ASME Journal of Mechanical Design, 124(1):47-51.

[2] Yang. G, and Chen, I-M, 2000, "Task-based optimization of modular robot configurations: minimized degree-of-freedom approach," Mechanism and Machine Theory 35:517-540.

[3] Herve, J M, 1999, "The Lie group of rigid body displacements, a fundamental tool for mechanism design," Mechanism and Machine Theory, 34:717-730.

[4] Wenger, P, 2000, "Some guidelines for the kinematic design of new manipulators," Mechanism and Machine Theory, 35:437-449

[5] Chablat, D, Wenger, P, Majou, F, and Merlet, J-P, 2004, "An Interval Analysis Based Study for the Design and the Comparison of Three-Degrees-of-Freedom Parallel Kinematic Machines," The International Journal of Robotics Research, 23(6): 615-624.

[6] Li, Z, Li, M, Chetwynd, D G, and Gosselin, C M, 2004, "Conceptual Design and Dimensional Synthesis of novel 2-DOF Translational Parallel Robot for Pick and Place Operations," ASME Journal of Mechanical Design, 126(3):449-455.

[7] Lee, E., and Mavroidis, D., 2002, "Solving the Geometric Design Problem of Spatial 3R Robot Manipulators Using Polynomial Homotopy Continuation," J. Mechanical Design, 124(4):652-661.

[8] Lee, E, and Mavroidis, C, 2004, "Geometric Design of 3R Manipulators for Reaching Four End-Effector Spatial Poses," The International Journal of Robotics Research, 23(3): 247-254.

[9] Suh, C. H., 1969, "On the Duality in the Existence of RR Links for Three Positions," ASME Journal of Engineering for Industry, 91B:129-134.

[10] Tsai, L. W., and Roth, B., 1972, "Design of Dyads with Helical, Cylindrical, Spherical, Revolute and Prismatic Joints," Mechanism and Machine Theory, 7:591-598.

[11] Perez, A, and McCarthy, J M, 2004a, "Geometric Design of RRP, RPR and PRR Serial Chains," Mechanism and Machine Theory, (in press)

[12] Suh, C.H. and Radcliffe, C.W., 1978, Kinematics and Mechanisms Design, John Wiley \& Sons, New York.

[13] McCarthy, J. M., 2000, Geometric Design of Linkages, SpringerVerlag, New York.

[14] Chen, P. and Roth, B., 1967, "Design Equations for Finitely and Infinitesimally Separated Position Synthesis of Binary Link and Combined Link Chains," ASME Journal of Engineering for Industry 91:209-219.

[15] Su, H, McCarthy, J M, and Watson, L T, 2004, "Generalized Linear Product Homotopy Algorithms and the Computation of Reachable Surfaces," ASME Journal of Computers and Information Science and Engineering, (in press).

[16] Craig, J. J., 1989, Introduction to Robotics, Mechanics and Control, Addison Wesley Publ. Co.

[17] Tsai, L-W, 1999, Robot Analysis: The Mechanics of Serial and Parallel Manipulators, Interscience, New York.

[18] McCarthy, J. M., 1990, Introduction to Theoretical Kinematics, The MIT Press.

[19] Murray, R. M., Sastry, S. S., and Li, Z., 1994, A Mathematical Introduction to Robotic Manipulation, CRC Press, Inc., Boca Raton, FL, 480pp.

[20] Gupta, K. C., 1986, "Kinematic analysis of manipulators using the zero reference position description," International Journal of Robotics Research, 5(2):5-13.

[21] Mullineux, G, 2004, "Modeling Spatial Displacements Using Clifford Algebra, ASME Journal of Mechanical Design 124(3):420-424.

[22] Daniilidis, K, 1999, "Hand-Eye Calibration Using Dual Quaternions," International Journal of Robotics Research, 18(3):286-298.

[23] Perez, A, and McCarthy, J M, 2004b, "Dual Quaternion Synthesis of Constrained Robotic Systems," ASME Journal of Mechanical Design, 126(3):425-435.

[24] Perez, A., 2003, "Dual Quaternion Synthesis of Constrained Robotic Systems", Ph.D. Dissertation, University of California, Irvine, September 2003.

[25] Castelain, J.M., Flamme, J.M., Gorla, B., and Renaud, M., "Computation of the direct and inverse geometric and differential models of robot manipulators with the aid of the hypercomplex theory", 8 th Int. Conference on Industrial Robot Technology, Brussels, 1986.

[26] Crane, C. D., and Duffy, J., 1998, Kinematic Analysis of Robot Manipulators, Cambridge University Press, 429pp. 